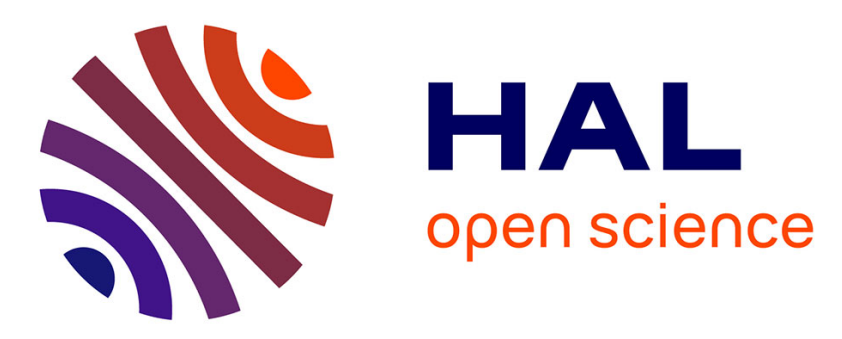

\title{
An analytical derivation for second-order blind separation of two signals
}

Abdelfettah Meziane Bentahar Meziane, Thierry Chonavel, Abdeldjalil Aissa El Bey, Adel Belouchrani

\section{- To cite this version:}

Abdelfettah Meziane Bentahar Meziane, Thierry Chonavel, Abdeldjalil Aissa El Bey, Adel Belouchrani. An analytical derivation for second-order blind separation of two signals. Annals of Telecommunications - annales des télécommunications, 2018, 73 (11), pp.711 - 717. 10.1007/s12243018-0635-6 . hal-01770936

\section{HAL Id: hal-01770936 https://hal.science/hal-01770936}

Submitted on 19 Apr 2018

HAL is a multi-disciplinary open access archive for the deposit and dissemination of scientific research documents, whether they are published or not. The documents may come from teaching and research institutions in France or abroad, or from public or private research centers.
L'archive ouverte pluridisciplinaire HAL, est destinée au dépôt et à la diffusion de documents scientifiques de niveau recherche, publiés ou non, émanant des établissements d'enseignement et de recherche français ou étrangers, des laboratoires publics ou privés. 


\title{
An analytical derivation for second-order blind
}

\section{separation of two signals}

\author{
Abdelfettah Meziane Bentahar Meziane \\ Thierry Chonavel · Abdeldjalil \\ Aïssa-El-Bey · Adel Belouchrani
}

Received: 15 October 2017 / Accepted: date

\begin{abstract}
In this paper, we propose analytical formulas that involve second-order statistics for separating two signals. The method utilizes source decorrelation and correlation function diversity. In particular, the proposed SOBAS (SecondOrder Blind Analytical Separation) algorithm differs from the ASOBI (Analytical Second-Order Blind Identification) algorithm in that it does not require prior knowledge or estimation of the noise variance. Computer simulations demonstrate the effectiveness of the proposed method.
\end{abstract}

\footnotetext{
A. Meziane Bentahar Meziane · A. Belouchrani

Ecole Nationale Polytechnique, Avenue Hacen Badi El harrach, 16200 Algiers, Algeria

E-mail: abdelfettah.meziane@enp.edu.dz

E-mail: adel.belouchrani@enp.edu.dz
}

T. Chonavel · A. Aïssa-El-Bey

IMT Atlantique, UMR CNRS 6285 Lab-STICC, UBL, F-29238 Brest Cedex, France

E-mail: thierry.chonavel@imt-atlantique.fr

E-mail: abdeldjalil.aissaelbey@imt-atlantique.fr 
Keywords Blind source separation · second-order statistics · TITO systems.

\section{Introduction}

Blind source separation (BSS) aims to identify a linear system from the observation of its output. When an array of sensors samples the field radiated by narrow-band sources, its output is classically modeled as an instantaneous spatial mixture of several independent sources, possibly corrupted by additive noise. Source separation can be achieved by first identifying the directional vectors associated with each source and then identifying source signals. In contrast with classical array processing, blind source separation performs identification without resorting to the knowledge of the array manifold. Hence, blind source separation is essentially unaffected by errors in the propagation model or in array calibration.

The term blind refers to a wide class of problems in signal and image processing, where one needs to extract the underlying sources from a set of observed mixtures. Almost no prior knowledge exists about the sources, nor about the mixing, hence the name blind. The mixing can be linear, nonlinear, instantaneous or convolutive. BSS is now a well-established area in Signal Processing with solid theoretical foundations and many potential applications.

In fact, BSS has become a very important topic of research and development in many areas, especially biomedical engineering and medical imaging [6, 10, speech and audio processing [2, 3], remote sensing [8, communications systems [1,7] and radar processing [1].

The particular case of two-inputs-two-outputs (TITO) systems is addressed in the literature for applications such as separation of speech sources [12. In 
[5] the TITO ASOBI (Analytical Second-Order Blind Identification) algorithm is proposed. It supplies a closed-form solution to the BSS problem. However, in [5], it is assumed that the noise variance is known.

In this paper, we propose a closed-form solution for the two-inputs-two-outputs BSS problem that differs from the one in [5]. In particular, our approach, referred to as SOBAS (Second-Order Blind Analytical Separation), works without prior knowledge (or estimation) of the noise variance. One main difference between ASOBI and SOBAS is that the former uses an average of correlations at different lags, including the zero lag, while the latter fully exploits the vector of non-zero lag correlations.

The paper is organized as follows. In section II, we describe the ASOBI algorithm. In section III, the proposed SOBAS is introduced. In section IV, simulations show the good behavior of SOBAS, and section V presents conclusions.

\section{Background: Analytical Solution for Second-Order Blind Identification (ASOBI) Algorithm}

In this section, we briefly recall the analytical solution for second-order blind identification (ASOBI) presented in [5] and introduce some notations. Let us consider an array of two sensors receiving two stationary, mutually uncorrelated and zero mean signals. The array outputs, denoted by $x_{i}(t)(i=1,2)$, form a $2 \times 1$ random vector. They are corrupted by additive white noise, denoted by $\boldsymbol{n}(t)$. Then, letting $s_{i}(t)$ denote the $i^{\text {th }}$ source signal and $x_{j}(t)$ the observation on the $j^{\text {th }}$ sensor, the model for the observed vector at discrete time $t(t=1, \ldots, T-1)$ is given by

$$
\boldsymbol{x}(t)=\boldsymbol{A} \boldsymbol{s}(t)+\boldsymbol{n}(t),
$$


where $\boldsymbol{s}(t)=\left[s_{1}(t) s_{2}(t)\right]^{T}$ and $\boldsymbol{x}(t)=\left[x_{1}(t) x_{2}(t)\right]^{T}$. Entry $(i, j)$ of the $2 \times 2$ unknown channel matrix $\boldsymbol{A}$ will be denoted by $a_{i j}$. Note that, in the same way, $m_{i j}$ will denote entry $(i, j)$ of any given matrix M. $\boldsymbol{n}(t)$ is a white noise process with covariance matrix $\sigma^{2} \mathbf{I}_{2}$, where $\mathbf{I}_{2}$ is the $2 \times 2$ identity matrix. The purpose of blind source separation is to recover the source signals from the array output $\boldsymbol{x}(t)$ without any prior knowledge about the mixture matrix $\boldsymbol{A}$. Source separation techniques based on second-order statistics assume uncorrelated source signals. In a blind context, it is well known that $\boldsymbol{A}$ can only be identified up to a permutation and multiplication by a scaling of its columns. Source indexing and energy are then transformed accordingly, that is, in such a way that observation $\boldsymbol{x}(t)$ remains unchanged [6. The crucial point is that these indeterminacies do not impede source separation.

From observations $\left\{x_{i}(t)\right\}_{t=0, \ldots, T-1}(i=1,2)$, we will need to calculate covariances among vectors in the form

$-\boldsymbol{x}_{i, t}=\left[x_{i}(t), \ldots, x_{i}(t+N-1)\right]^{T}, t=0, \ldots T-N$,

where superscript $(\cdot)^{T}$ denotes the transpose of a vector and $N$ is the number of correlation lags that will be involved in computations. We also introduce the following notations:

$-s_{i, t}=\left[s_{i}(t), \ldots, s_{i}(t+N-1)\right]^{T}$,

- $\boldsymbol{R}_{\boldsymbol{s}_{i} \boldsymbol{s}_{j}}=\mathbb{E}\left[\boldsymbol{s}_{i, t} \boldsymbol{s}_{j, t}^{T}\right]$,

$-\boldsymbol{R}_{\boldsymbol{x}_{i} \boldsymbol{x}_{j}}=\mathbb{E}\left[\boldsymbol{x}_{i, t} \boldsymbol{x}_{j, t}^{T}\right]$ 
where $\mathbb{E}[\cdot]$ denotes the expectation operator. Then we get

$$
\begin{aligned}
& \boldsymbol{R}_{\boldsymbol{x}_{1} \boldsymbol{x}_{1}}=a_{11}^{2} \boldsymbol{R}_{\boldsymbol{s}_{1} \boldsymbol{s}_{1}}+a_{12}^{2} \boldsymbol{R}_{\boldsymbol{s}_{2} \boldsymbol{s}_{2}}+\sigma^{2} \boldsymbol{I}_{N} \\
& \boldsymbol{R}_{\boldsymbol{x}_{2} \boldsymbol{x}_{2}}=a_{21}^{2} \boldsymbol{R}_{\boldsymbol{s}_{1} \boldsymbol{s}_{1}}+a_{22}^{2} \boldsymbol{R}_{\boldsymbol{s}_{2} \boldsymbol{s}_{2}}+\sigma^{2} \boldsymbol{I}_{N} \\
& \boldsymbol{R}_{\boldsymbol{x}_{1} \boldsymbol{x}_{2}}=a_{11} a_{21} \boldsymbol{R}_{\boldsymbol{s}_{1} \boldsymbol{s}_{1}}+a_{12} a_{22} \boldsymbol{R}_{\boldsymbol{s}_{2} \boldsymbol{s}_{2}} .
\end{aligned}
$$

We assume that $T$ vectors $\mathbf{x}(t)$ are available. Then, letting $T=N K$ where $K$ represents the number of non-overlapping vectors $\boldsymbol{x}_{i, t}, \boldsymbol{R}_{\boldsymbol{x}_{i} \boldsymbol{x}_{j}}$ can be estimated by

$$
\widehat{\boldsymbol{R}}_{\boldsymbol{x}_{i} \boldsymbol{x}_{j}}=\frac{1}{K} \sum_{k=0}^{K-1} \boldsymbol{x}_{i, N k} \boldsymbol{x}_{j, N k}^{T}
$$

Alternatively, one could average overlapping vectors to get a more precise estimate of $\boldsymbol{R}_{\boldsymbol{x}_{i} \boldsymbol{x}_{j}}$, but at the expense of higher computational complexity. To track possible non-stationarity, these correlation matrix estimates can be updated adaptively. Operators $\overline{\operatorname{tr}}(\cdot)$ and $\overline{\text { off }}(\cdot)$ calculate means of diagonal and off-diagonal entries of a matrix, respectively. Then, if $\boldsymbol{G}$ is any square matrix of size $N \times N$,

$$
\begin{aligned}
& \overline{\mathrm{off}}(\boldsymbol{G})=\frac{1}{N(N-1)} \sum_{i=1}^{N} \sum_{\substack{j=1 \\
j \neq i}}^{N} g_{i j} \\
& \overline{\operatorname{tr}}(\boldsymbol{G})=\frac{1}{N} \sum_{i=1}^{N} g_{i i} .
\end{aligned}
$$


By applying these operators to equation (2), we get the following set of relations:

$$
\begin{aligned}
& F_{1}=\overline{\operatorname{off}}\left(\boldsymbol{R}_{\boldsymbol{x}_{1} \boldsymbol{x}_{1}}\right)=a_{11}^{2} R_{1}+a_{12}^{2} R_{2} \\
& F_{2}=\overline{\operatorname{off}}\left(\boldsymbol{R}_{\boldsymbol{x}_{2} \boldsymbol{x}_{2}}\right)=a_{21}^{2} R_{1}+a_{22}^{2} R_{2} \\
& F_{12}=\overline{\operatorname{off}}\left(\boldsymbol{R}_{\boldsymbol{x}_{1} \boldsymbol{x}_{2}}\right)=a_{11} a_{21} R_{1}+a_{12} a_{22} R_{2} \\
& T_{1}=\overline{\operatorname{tr}}\left(\boldsymbol{R}_{\boldsymbol{x}_{1} \boldsymbol{x}_{1}}\right)=a_{11}^{2}+a_{12}^{2}+\sigma^{2} \\
& T_{2}=\overline{\operatorname{tr}}\left(\boldsymbol{R}_{\boldsymbol{x}_{2} \boldsymbol{x}_{2}}\right)=a_{21}^{2}+a_{22}^{2}+\sigma^{2} \\
& T_{12}=\overline{\operatorname{tr}}\left(\boldsymbol{R}_{\boldsymbol{x}_{1} \boldsymbol{x}_{2}}\right)=a_{11} a_{21}+a_{12} a_{22},
\end{aligned}
$$

where $R_{i}=\overline{\mathrm{off}}\left(\boldsymbol{R}_{\boldsymbol{s}_{i} \boldsymbol{s}_{i}}\right), i=1,2$. In the last 3 equations of $(5)$, we have used the fact that, under the assumption of unit-variance source signals, $\overline{\operatorname{tr}}\left(\boldsymbol{R}_{\boldsymbol{s}_{i} \boldsymbol{s}_{i}}\right)=1, i=1,2$. By solving equations (5), we obtain the following estimate for the mixing matrix [5]:

$$
\widehat{\boldsymbol{A}}=\left[\begin{array}{cc}
\sqrt{\frac{F_{1}-\left(T_{1}-\sigma^{2}\right) \beta}{\gamma}} & \frac{\left(F_{12}-T_{12} \alpha\right) \sqrt{\gamma}}{\gamma \sqrt{\left(T_{2}-\sigma^{2}\right) \alpha-F_{2}}} \\
\frac{\left(T_{12} \beta-F_{12}\right) \sqrt{\gamma}}{\gamma \sqrt{F_{1}-\left(T_{1}-\sigma^{2}\right) \beta}} & \sqrt{\frac{\left(T_{2}-\sigma^{2}\right) \alpha-F_{2}}{\gamma}}
\end{array}\right],
$$

where $\alpha, \beta$ and $\gamma$ are given in Algorithm 1. The ASOBI algorithm is summarized in Algorithm 1 . 
Algorithm 1 ASOBI Algorithm

Require: Observed signal $\boldsymbol{x}(t)$ and the noise variance $\sigma^{2}$.

Ensure: The mixing matrix $\boldsymbol{A}$ and source signals $\boldsymbol{s}(t)$.

1: Estimate the matrices $\widehat{\boldsymbol{R}}_{\boldsymbol{x}_{i} \boldsymbol{x}_{j}}=\frac{1}{K} \sum_{k=0}^{K-1} \boldsymbol{x}_{i, N k} \boldsymbol{x}_{j, N k}^{T}$ for $i=1,2$ and $j=1,2$;

2: Compute the coefficients $F_{i}=\overline{\operatorname{off}}\left(\widehat{\boldsymbol{R}}_{\boldsymbol{x}_{i} \boldsymbol{x}_{i}}\right)$ for $i=1,2$ and $F_{12}=\overline{\operatorname{off}}\left(\widehat{\boldsymbol{R}}_{\boldsymbol{x}_{1} \boldsymbol{x}_{2}}\right)$;

3: Compute the coefficients $T_{i}=\overline{\operatorname{tr}}\left(\widehat{\boldsymbol{R}}_{\boldsymbol{x}_{i} \boldsymbol{x}_{i}}\right)$ for $i=1,2$ and $T_{12}=\overline{\operatorname{tr}}\left(\widehat{\boldsymbol{R}}_{\boldsymbol{x}_{1} \boldsymbol{x}_{2}}\right)$;

4: Compute the coefficients

$$
\begin{aligned}
a= & 2 F_{12} T_{12}-\left(F_{1}\left(T_{2}-\sigma^{2}\right)+F_{2}\left(T_{1}-\sigma^{2}\right)\right) \\
b= & 2\left(T_{12}^{2}-\left(T_{1}-\sigma^{2}\right)\left(T_{2}-\sigma^{2}\right)\right) \\
c^{2}= & \left(F_{1}\left(T_{2}-\sigma^{2}\right)-F_{2}\left(T_{1}-\sigma^{2}\right)\right)^{2}+ \\
& \left.4\left(F_{12}\left(T_{2}-\sigma^{2}\right)-T_{12} F_{2}\right)\left(F_{12}\left(T_{1}-\sigma^{2}\right)-T_{12} F_{1}\right)\right) ;
\end{aligned}
$$

5: Compute the coefficients $\alpha=(a+c) / b, \beta=(a-c) / b$ and $\gamma=2 c / b$;

6: Estimate the matrix $\widehat{\boldsymbol{A}}=\left[\begin{array}{ll}\sqrt{\frac{F_{1}-\left(T_{1}-\sigma^{2}\right) \beta}{\gamma}} & \frac{\left(F_{12}-T_{12} \alpha\right) \sqrt{\gamma}}{\gamma \sqrt{\left(T_{2}-\sigma^{2}\right) \alpha-F_{2}}} \\ \frac{\left(T_{12} \beta-F_{12}\right) \sqrt{\gamma}}{\gamma \sqrt{F_{1}-\left(T_{1}-\sigma^{2}\right) \beta}} & \sqrt{\frac{\left(T_{2}-\sigma^{2}\right) \alpha-F_{2}}{\gamma}}\end{array}\right]$;

7: Estimate the source signals $\widehat{\boldsymbol{s}}(t)=\widehat{\boldsymbol{A}}^{-1} \boldsymbol{x}(t)$.

Let us note that an estimate of the noise variance $\sigma^{2}$ is needed to achieve estimation of the channel coefficients. It can be obtained by the eigen-decomposition of the data covariance matrix if a third sensor is available [5]. Alternatively, $\sigma^{2}$ can be estimated using only two sensors before data recording begins. It is also possible to estimate $\sigma^{2}$ by choosing the value that minimizes (in the least-squares sense w.r.t. $\sigma^{2}$ ) the intercorrelations (at different time lags) between the two outputs of $\boldsymbol{A}\left(\sigma^{2}\right)^{-1} \boldsymbol{x}(t)$. 


\section{Second-Order Blind Analytical Separation (SOBAS) Algorithm}

To avoid the requirement of prior knowledge of noise variance for estimating matrix

A and to better benefit from the diversity information contained in the covariance coefficients of source signals, we propose an alternative algorithm to deal with separation of 2 sources, named SOBAS (Second-Order Blind Analytical Separation). Instead of considering averaged correlation coefficients, as done in ASOBI, our solution involves all non-zero covariance lags. Removing the zero-lag coefficient makes it possible to work with noise-free statistics.

Note also that in ASOBI source separation cannot be achieved if the $R_{i}$, defined by $R_{i}=\overline{\operatorname{off}}\left(\boldsymbol{R}_{\boldsymbol{s}_{i} \boldsymbol{s}_{i}}\right)$ are equal. In SOBAS, we fully account for the covariance lags of signals instead of having them averaged, which results in more robustness against spectral similarity of sources.

In addition, in the literature ASOBI has been derived for the case of real valued signals, while we shall derive SOBAS in the more general context of complex signals. Then, the entries of the mixing matrix are complex valued. In the following $\boldsymbol{R}_{\boldsymbol{x}_{i} \boldsymbol{x}_{j}}$ will stand for $\mathbb{E}\left[\boldsymbol{x}_{i, t} \boldsymbol{x}_{j, t}^{H}\right]$, where exponent $(\cdot)^{H}$ stands for the transposeconjugate operator. Similarly, we will have $\boldsymbol{R}_{\boldsymbol{s}_{i} \boldsymbol{s}_{i}}=\mathbb{E}\left[\boldsymbol{s}_{i, t} \boldsymbol{s}_{i, t}^{H}\right]$ and the extension of Eq. 22 to the complex case can be written as

$$
\boldsymbol{R}_{\boldsymbol{x}_{i} \boldsymbol{x}_{j}}=a_{i 1} a_{j 1}^{*} \boldsymbol{R}_{\boldsymbol{s}_{1} \boldsymbol{s}_{1}}+a_{i 2} a_{j 2}^{*} \boldsymbol{R}_{\boldsymbol{s}_{2} \boldsymbol{s}_{2}}+\delta_{i j} \sigma^{2} \boldsymbol{I}_{N} \quad(i, j=1,2),
$$

where $(\cdot)^{*}$ stands for complex conjugation and $\delta_{i j}=1$ if $i=j$ and 0 otherwise.

Furthermore, unlike in the SOBI algorithm [4, the implementation that we propose here does not require whitening. After whitening, the mixing matrix identification only amounts to a rotation identification, which requires little computational ef- 
fort. However, this operation relies on a noise-free approximation, which slightly degrades performance.

To derive the SOBAS algorithm, we denote by offc $(\boldsymbol{G})$ the first column vector of matrix $\boldsymbol{G}$ with its first entry $g_{11}$ removed:

$$
\operatorname{offc}(\boldsymbol{G})=\left[g_{21}, \ldots, g_{N 1}\right]^{T}
$$

For $i, j=1,2$ we get

$$
\begin{aligned}
& \boldsymbol{f}_{i j}=\operatorname{offc}\left(\boldsymbol{R}_{\boldsymbol{x}_{i} \boldsymbol{x}_{j}}\right) \\
& \boldsymbol{r}_{i}=\operatorname{offc}\left(\boldsymbol{R}_{\boldsymbol{s}_{i} \boldsymbol{s}_{i}}\right) .
\end{aligned}
$$

Then, applying the offc $(\cdot)$ operator to Eq. (7) yields

$$
\begin{aligned}
& \boldsymbol{f}_{11}=\operatorname{offc}\left(\boldsymbol{R}_{\boldsymbol{x}_{1} \boldsymbol{x}_{1}}\right)=\left|a_{11}\right|^{2} \boldsymbol{r}_{1}+\left|a_{12}\right|^{2} \boldsymbol{r}_{2} \\
& \boldsymbol{f}_{22}=\operatorname{offc}\left(\boldsymbol{R}_{\boldsymbol{x}_{2} \boldsymbol{x}_{2}}\right)=\left|a_{21}\right|^{2} \boldsymbol{r}_{1}+\left|a_{22}\right|^{2} \boldsymbol{r}_{2} \\
& \boldsymbol{f}_{12}=\operatorname{offc}\left(\boldsymbol{R}_{\boldsymbol{x}_{1} \boldsymbol{x}_{2}}\right)=a_{11} a_{21}^{*} \boldsymbol{r}_{1}+a_{12} a_{22}^{*} \boldsymbol{r}_{2} \\
& \boldsymbol{f}_{21}=\operatorname{offc}\left(\boldsymbol{R}_{\boldsymbol{x}_{2} \boldsymbol{x}_{1}}\right)=a_{11}^{*} a_{21} \boldsymbol{r}_{1}+a_{12}^{*} a_{22} \boldsymbol{r}_{2},
\end{aligned}
$$

where |.| stands for the complex modulus. Note that in Eq. (9), all terms are independent from the noise variance $\sigma^{2}$.

The system of equation (9) can be rewritten as

$$
\begin{aligned}
& {\left[\begin{array}{ll}
\boldsymbol{f}_{11} & \boldsymbol{f}_{12}
\end{array}\right]=\left[\begin{array}{ll}
\boldsymbol{r}_{1} & \boldsymbol{r}_{2}
\end{array}\right] \boldsymbol{D}_{1} \boldsymbol{A}^{H}} \\
& {\left[\begin{array}{lll}
\boldsymbol{f}_{21} & \boldsymbol{f}_{22}
\end{array}\right]=\left[\begin{array}{ll}
\boldsymbol{r}_{1} & \boldsymbol{r}_{2}
\end{array}\right] \boldsymbol{D}_{2} \boldsymbol{A}^{H},}
\end{aligned}
$$

where $\boldsymbol{D}_{1}=\left[\begin{array}{cc}a_{11} & 0 \\ 0 & a_{12}\end{array}\right]$ and $\boldsymbol{D}_{2}=\left[\begin{array}{cc}a_{21} & 0 \\ 0 & a_{22}\end{array}\right]$. Matrix $\boldsymbol{A}$ is assumed to be invertible and since blind source separation relies on a spectral diversity assumption about sources, matrix $\left[\boldsymbol{r}_{1} \boldsymbol{r}_{2}\right]$ should be rank two. To recover $\boldsymbol{A}$, we assume first that $\boldsymbol{D}_{2}$ is invertible, that is $\operatorname{rank}\left(\left[\boldsymbol{f}_{21} \boldsymbol{f}_{22}\right]\right)=2$. Then,

$$
\left[\boldsymbol{f}_{21} \boldsymbol{f}_{22}\right]^{\#}\left[\boldsymbol{f}_{11} \boldsymbol{f}_{12}\right] \boldsymbol{A}^{-H}=\boldsymbol{A}^{-H} \widetilde{\boldsymbol{D}}
$$


with $\widetilde{\boldsymbol{D}}=\boldsymbol{D}_{2}^{-1} \boldsymbol{D}_{1}$ and $(\cdot)^{\#}$ is pseudo-inverse matrix operator such that $\left[\boldsymbol{f}_{21} \boldsymbol{f}_{22}\right]^{\#}=$ $\left(\left[\boldsymbol{f}_{21} \boldsymbol{f}_{22}\right]^{H}\left[\boldsymbol{f}_{21} \boldsymbol{f}_{22}\right]\right)^{-1}\left[\boldsymbol{f}_{21} \boldsymbol{f}_{22}\right]^{H}$. Problem 120 is an eigenvector problem where

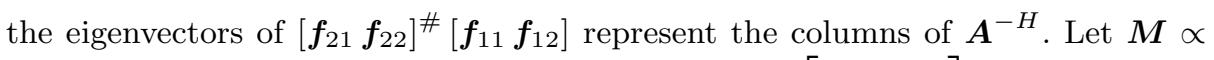
$\left[\begin{array}{ll}\boldsymbol{f}_{21} & \boldsymbol{f}_{22}\end{array}\right]^{\#}\left[\begin{array}{ll}\boldsymbol{f}_{11} & \boldsymbol{f}_{12}\end{array}\right]$ be a $2 \times 2$ matrix such that $\boldsymbol{M}=\left[\begin{array}{cc}m_{11} & m_{12} \\ m_{21} & m_{22}\end{array}\right]$ where

$$
\begin{aligned}
& m_{11}=\left\|\boldsymbol{f}_{22}\right\|_{2}^{2}\left(\boldsymbol{f}_{21}^{H} \boldsymbol{f}_{11}\right)-\left(\boldsymbol{f}_{21}^{H} \boldsymbol{f}_{22}\right)\left(\boldsymbol{f}_{22}^{H} \boldsymbol{f}_{11}\right) \\
& m_{12}=\left\|\boldsymbol{f}_{22}\right\|_{2}^{2}\left(\boldsymbol{f}_{21}^{H} \boldsymbol{f}_{12}\right)-\left(\boldsymbol{f}_{21}^{H} \boldsymbol{f}_{22}\right)\left(\boldsymbol{f}_{22}^{H} \boldsymbol{f}_{12}\right) \\
& m_{21}=\left\|\boldsymbol{f}_{21}\right\|_{2}^{2}\left(\boldsymbol{f}_{22}^{H} \boldsymbol{f}_{11}\right)-\left(\boldsymbol{f}_{22}^{H} \boldsymbol{f}_{21}\right)\left(\boldsymbol{f}_{21}^{H} \boldsymbol{f}_{11}\right) \\
& m_{22}=\left\|\boldsymbol{f}_{21}\right\|_{2}^{2}\left(\boldsymbol{f}_{22}^{H} \boldsymbol{f}_{12}\right)-\left(\boldsymbol{f}_{22}^{H} \boldsymbol{f}_{21}\right)\left(\boldsymbol{f}_{21}^{H} \boldsymbol{f}_{12}\right) .
\end{aligned}
$$

Then, we can estimate $\boldsymbol{A}^{-H}$ as:

$$
\boldsymbol{A}^{-H}=\left[\begin{array}{cc}
m_{12} & m_{12} \\
\lambda_{1}-m_{11} & \lambda_{2}-m_{11}
\end{array}\right],
$$

where

$$
\begin{aligned}
& \lambda_{1}=\frac{1}{2}\left(m_{11}+m_{22}+\sqrt{\left(m_{11}+m_{22}\right)^{2}-4\left(m_{11} m_{22}-m_{12} m_{21}\right)}\right) \\
& \lambda_{2}=\frac{1}{2}\left(m_{11}+m_{22}-\sqrt{\left(m_{11}+m_{22}\right)^{2}-4\left(m_{11} m_{22}-m_{12} m_{21}\right)}\right) .
\end{aligned}
$$

Now, if $\boldsymbol{D}_{2}$ is not invertible and $\boldsymbol{D}_{1}$ is invertible the same procedure can be applied by taking $\boldsymbol{M} \propto\left[\begin{array}{ll}\boldsymbol{f}_{11} & \boldsymbol{f}_{12}\end{array}\right]^{\#}\left[\boldsymbol{f}_{21} \boldsymbol{f}_{22}\right]$. If both $\boldsymbol{D}_{1}$ and $\boldsymbol{D}_{2}$ are not invertible, since $\boldsymbol{A}$ is invertible we get $\left(a_{11}, a_{22}\right)=(0,0)$ or $\left(a_{12}, a_{21}\right)=(0,0)$ and thus no separation is needed. As noted before, rank properties upon $\boldsymbol{D}_{1}$ and $\boldsymbol{D}_{2}$ are immediately available since $\operatorname{rank}\left(\boldsymbol{D}_{1}\right)=\operatorname{rank}\left(\left[\boldsymbol{f}_{11} \boldsymbol{f}_{12}\right]\right)$ and $\operatorname{rank}\left(\boldsymbol{D}_{2}\right)=\operatorname{rank}\left(\left[\boldsymbol{f}_{21} \boldsymbol{f}_{22}\right]\right)$.

The SOBAS algorithm is summarized in Algorithm 2 . 
Algorithm 2 SOBAS Algorithm

Require: Observed signal $\boldsymbol{x}(t)$.

Ensure: The mixing matrix $\boldsymbol{A}$ and source signals $\boldsymbol{s}(t)$.

1: Estimate the matrices $\widehat{\boldsymbol{R}}_{\boldsymbol{x}_{i} \boldsymbol{x}_{j}}=\frac{1}{K} \sum_{k=0}^{K-1} \boldsymbol{x}_{i, N k} \boldsymbol{x}_{j, N k}^{T}$ for $i=1,2$ and $j=1,2$;

2: Compute the vectors $\boldsymbol{f}_{i j}=\operatorname{offc}\left(\widehat{\boldsymbol{R}}_{\boldsymbol{x}_{i} \boldsymbol{x}_{j}}\right)$ for $i=1,2$ and $j=1,2$;

3: Compute $\varepsilon_{1}=\frac{\left|\boldsymbol{f}_{11}^{H} \boldsymbol{f}_{12}\right|}{\left\|\boldsymbol{f}_{11}\right\|_{2}\left\|\boldsymbol{f}_{12}\right\|_{2}}$ and $\varepsilon_{2}=\frac{\left|\boldsymbol{f}_{22}^{H} \boldsymbol{f}_{21}\right|}{\left\|\boldsymbol{f}_{22}\right\|_{2}\left\|\boldsymbol{f}_{21}\right\|_{2}}$;

4: if $\varepsilon_{1}<\varepsilon_{2}$ then

5: $\quad$ Compute the matrix $\boldsymbol{M}=\left[\begin{array}{ll}\boldsymbol{f}_{11} & \boldsymbol{f}_{12}\end{array}\right]^{\#}\left[\boldsymbol{f}_{21} \boldsymbol{f}_{22}\right]$;

6: else

7: $\quad$ Compute the matrix $M=\left[\begin{array}{ll}f_{21} & f_{22}\end{array}\right]^{\#}\left[f_{11} f_{12}\right]$;

8: end if

9: Estimate the matrix $\widehat{\boldsymbol{A}}^{-1}=\left[\begin{array}{ll}m_{12}^{*} & \lambda_{1}^{*}-m_{11}^{*} \\ m_{12}^{*} & \lambda_{2}^{*}-m_{11}^{*}\end{array}\right]$ with

$$
\begin{aligned}
& \lambda_{1}=\frac{1}{2}\left(m_{11}+m_{22}+\sqrt{\left(m_{11}+m_{22}\right)^{2}-4\left(m_{11} m_{22}-m_{12} m_{21}\right)}\right) \\
& \lambda_{2}=\frac{1}{2}\left(m_{11}+m_{22}-\sqrt{\left(m_{11}+m_{22}\right)^{2}-4\left(m_{11} m_{22}-m_{12} m_{21}\right)}\right) ;
\end{aligned}
$$

10: Estimate the source signals $\widehat{\boldsymbol{s}}(t)=\widehat{\boldsymbol{A}}^{-1} \boldsymbol{x}(t)$.

The numerical complexities of ASOBI and SOBAS are very close. It is the same in terms of multiplications: both require $\mathcal{O}\left(3(K+1) N^{2}\right)$ multiplications. It is slightly different for additions: ASOBI requires $\mathcal{O}\left(3(K+1) N^{2}\right)$ additions and SOBAS $\mathcal{O}\left(3 K N^{2}\right)$ additions.

\section{Simulation results}

To assess SOBAS performance, a comparison with SOBI [4] and ASOBI [5] is performed in the following situations: First, we assume that signals $s_{i}(t)(i=1,2)$ 
are $\mathrm{AR}(1)$ complex processes defined by difference equations in the form:

$$
s_{i}(t)=\phi_{i} s_{i}(t-1)+w_{i}(t) .
$$

The $w_{i}(i=1,2)$ are independent white noise with normal distributions. The AR coefficients $\phi_{i}(i=1,2)$ are generated such that $\phi_{i}=\varrho e^{j 2 \pi f_{i}}$. We set resonance frequencies equal to $f_{1}=0.02$ and $f_{2} \in\{0.04,0.10\}$. Note that the spacing of resonance frequencies is representative of dissimilarity between spectra. This can be checked by considering the Itakura-Saito distance [9]. In the particular case of AR(1) models, straightforward calculations show that this distance increases as a function of $f_{1}-f_{2}$ :

$$
d_{I S}\left(s_{1}, s_{2}\right)=\log \left(1+\frac{1+\varrho^{2}\left(1-2 \cos \left(2 \pi\left(f_{1}-f_{2}\right)\right)\right.}{1-\varrho^{2}}\right) .
$$

Performance levels are evaluated over $N_{k}=1000$ Monte-Carlo runs and estimation performance levels are given by the normalized mean-square error criterion :

$$
\mathrm{NMSE}=\frac{1}{N_{k}} \sum_{k=1}^{N_{k}} \sum_{i=1}^{2} 1-\left(\frac{\hat{\boldsymbol{s}}_{i, k}^{T} \boldsymbol{s}_{i}}{\left\|\widehat{\boldsymbol{s}}_{i, k}\right\|_{2}\left\|\boldsymbol{s}_{i}\right\|_{2}}\right)^{2},
$$

where $\widehat{\boldsymbol{s}}_{i, k}$ denotes the estimated source vector at the $k^{\text {th }}$ Monte-Carlo run. For these simulations, we compare the performance of SOBAS with the SOBI algorithm due to the fact that ASOBI was developed under the real signal assumption. In Figures 1 and 2 we fix $\varrho=0.9$ and we generate the complex mixing matrix $\boldsymbol{A}$ randomly at each Monte-Carlo run. From these figures, we can observe the superiority of SOBAS against the SOBI algorithm. Clearly, avoiding the use of statistics affected by noise variance enables significant NMSE performance improvement. In Figures 3 and 4 we fix the AR parameters $f_{1}=0.02, f_{2}=0.10$ and $\varrho=0.9$. We generate the mixing matrix $\boldsymbol{A}$ by using the following model $\boldsymbol{A}=\left[\begin{array}{cc}1 & 1 \\ e^{j \theta_{1}} & e^{j \theta_{2}}\end{array}\right]$ 


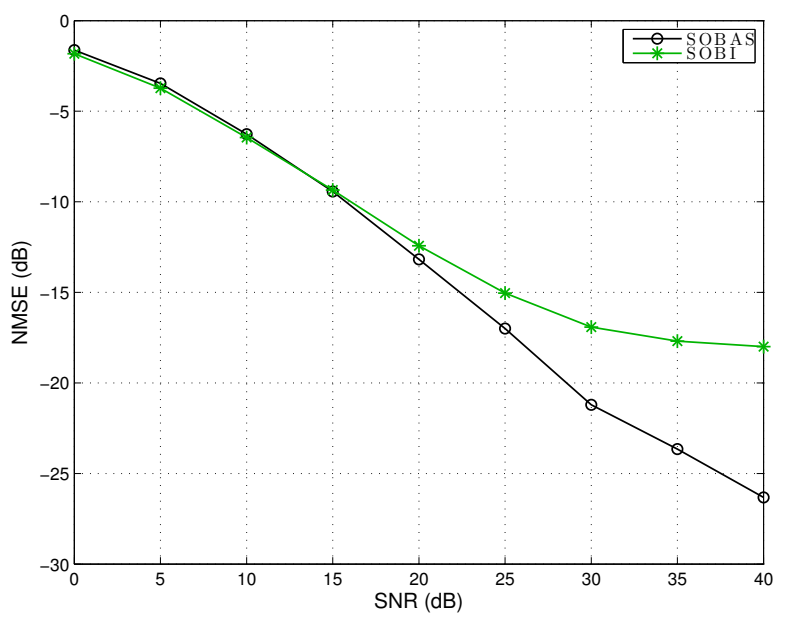

Fig. 1 Comparison of normalized mean-square error (NMSE) versus SNR for blind source separation system with $2 \mathrm{AR}(1)$ sources, 2 sensors, $T=1000, f_{1}=0.02, f_{2}=0.10$ and $\varrho=0.9$.

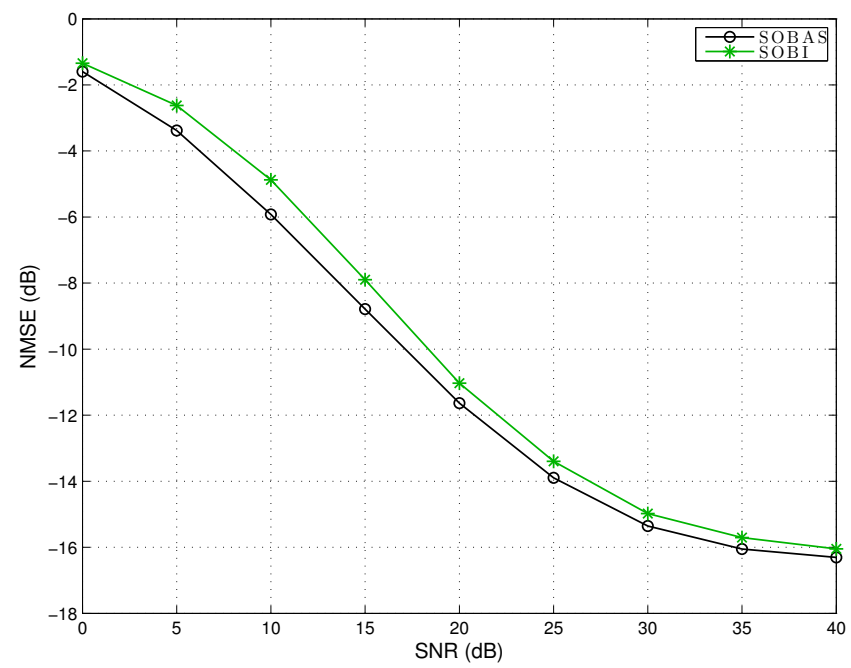

Fig. 2 Comparison of normalized mean-square error (NMSE) versus SNR for blind source separation system with $2 \mathrm{AR}(1)$ sources, 2 sensors, $T=1000, f_{1}=0.02, f_{2}=0.04$ and $\varrho=0.9$. 
such that $\theta_{1}=\frac{\pi}{12}$ and $\theta_{2}=\theta_{1}+\delta \theta$. As expected, NMSE performance improves as vector angle increases. SOBI shows limited performance for large angles, which can be explained by whitening matrix error and noise estimation error.

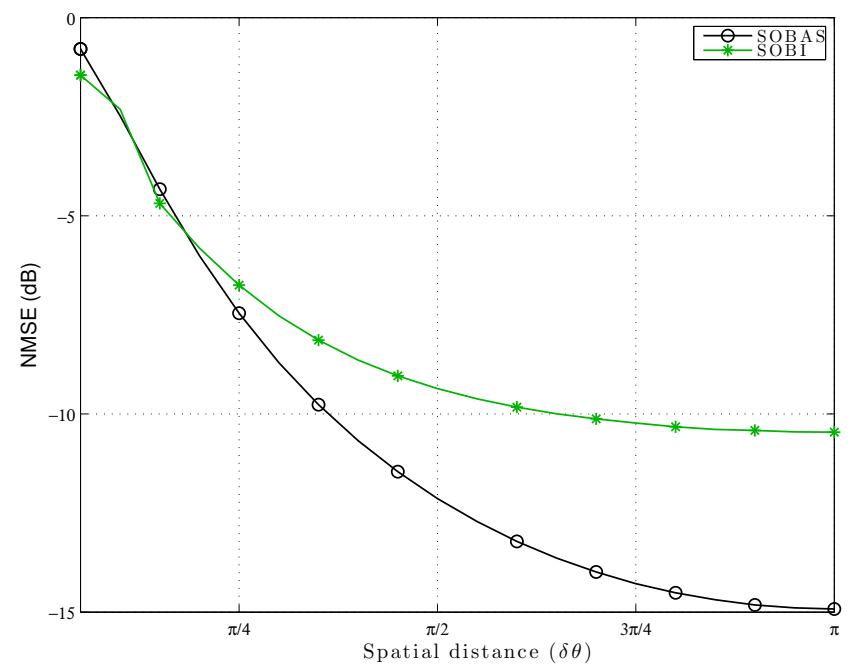

Fig. 3 Comparison of normalized mean-square error (NMSE) versus $\delta \theta$ for blind source separation system with $2 \mathrm{AR}(1)$ sources, 2 sensors, $T=1000, f_{1}=0.02, f_{2}=0.10, \varrho=0.9$ and $\mathrm{SNR}=15 \mathrm{~dB}$.

In Figures 5 and 6 we consider the blind separation of two audio sources sampled at $8 \mathrm{kHz}$. W generate the real mixing matrix $\boldsymbol{A}$ randomly. By applying the SOBAS algorithm, we obtain the results shown in Figure 5 at $\mathrm{SNR}=25 \mathrm{~dB}$ which represents the original sources, the two mixture signals and the audio sources estimated by the SOBAS algorithm, respectively. In Figure 6, we compare the performance of SOBAS with SOBI and ASOBI. For ASOBI we have consider the perfect knowledge the noise variance. This figure confirms the superiority of SOBAS against SOBI and ASOBI in the context of audio source separation. 


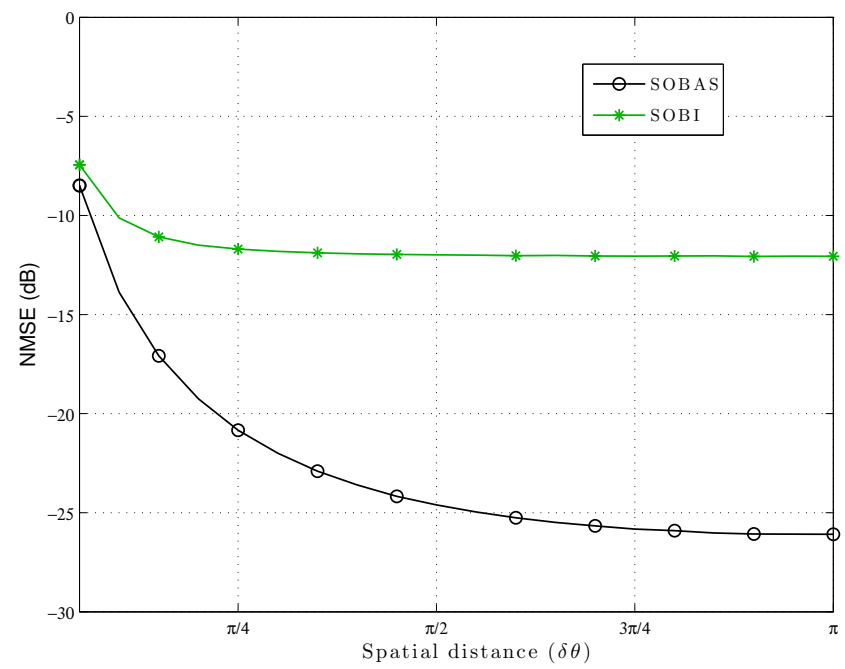

Fig. 4 Comparison of normalized mean-square error (NMSE) versus $\delta \theta$ for blind source separation system with $2 \mathrm{AR}(1)$ sources, 2 sensors, $T=1000, f_{1}=0.02, f_{2}=0.10, \varrho=0.9$ and $\mathrm{SNR}=30 \mathrm{~dB}$.

\section{Conclusions}

In this paper we have proposed an analytical formula for blind separation of a mixture of two sources observed by two sensors. The proposed SOBAS algorithm circumvents the assumption of known noise variance that was made in the ASOBI algorithm. In addition, it outperforms both SOBI and ASOBI in term of NMSE. Extensions of this approach to a higher number of sources and sensors will be worth addressing in further work. 

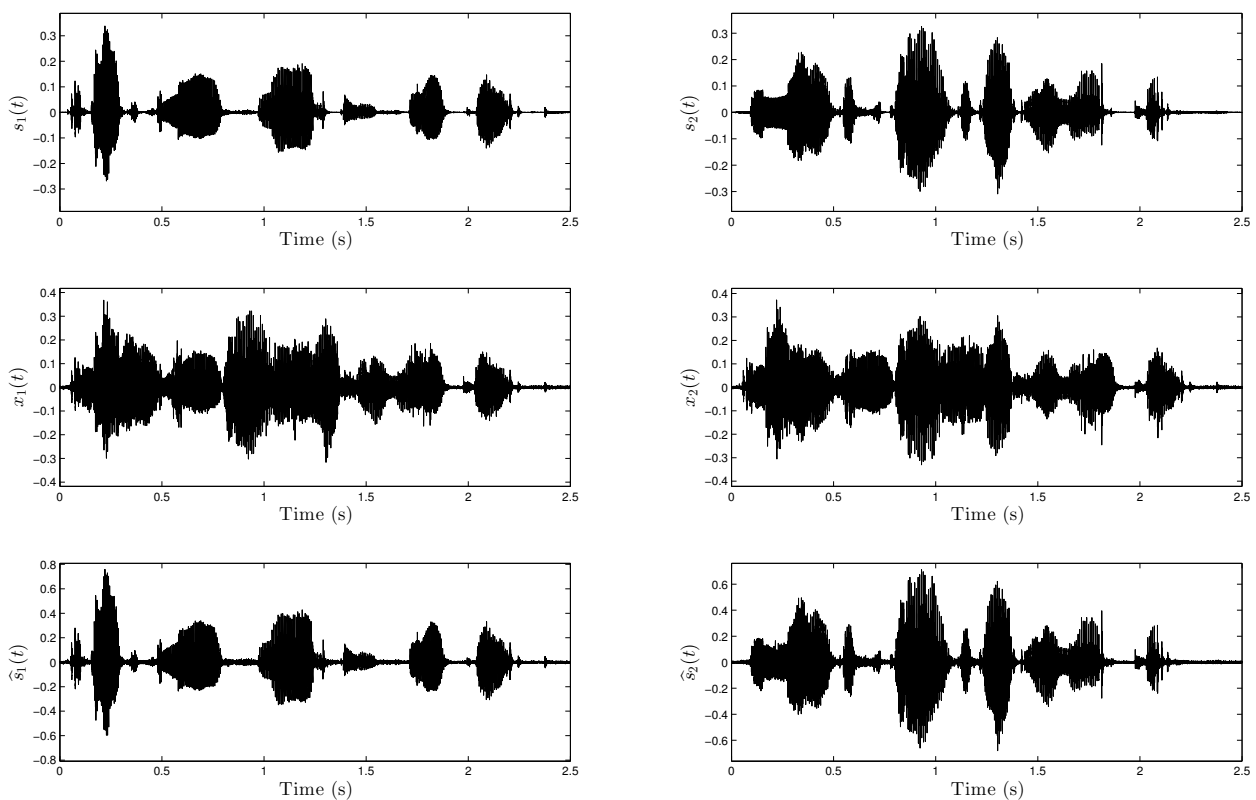

Fig. 5 Blind audio source separation of two sources from two mixtures by using SOBAS. Row 1: original sources; row 2: mixture signals; row 3: audio sources estimated by SOBAS.

\section{References}

1. Abed-Meraim, K., Attallah, S., Lim, T., Damen, M.: A blind interference canceller in DS-CDMA. In: IEEE International Symposium on Spread Spectrum Techniques and Applications, pp. 358-362. Parsippany (2000)

2. Aïssa-El-Bey, A., Abed-Meraim, K., Grenier, Y.: Underdetermined blind audio source separation using modal decomposition. EURASIP Journal on Audio, Speech, and Music Processing 2007(85438), 1-15 (2007)

3. Aziz-Sbaï, S.M., Aïssa-El-Bey, A., Pastor, D.: Contribution of statistical tests to sparseness-based blind source separation. EURASIP Journal on Applied Signal Processing 2012(169) (2012)

4. Belouchrani, A., Abed-Meraim, K., Cardoso, J.F., Moulines, E.: A blind source separation technique using second-order statistics. IEEE Transactions on Signal Processing 45(2), 


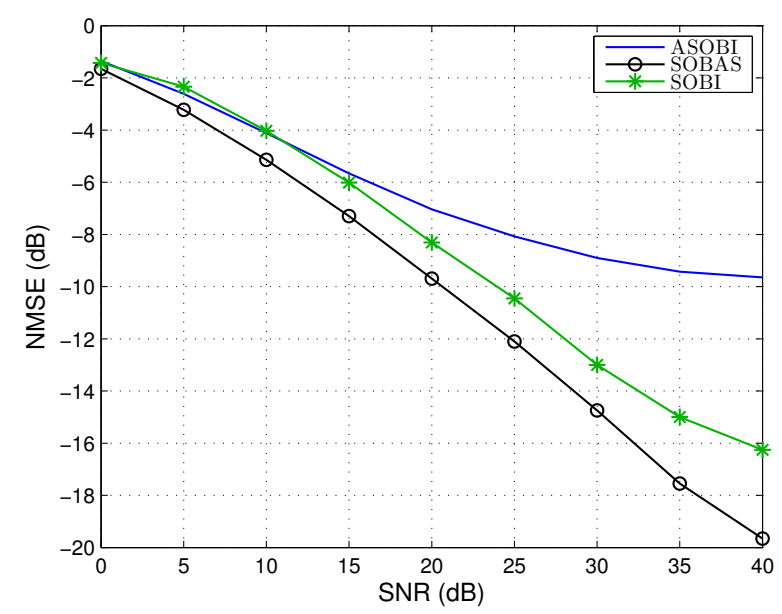

Fig. 6 Comparison of normalized mean-square error (NMSE) versus the SNR for blind source separation system with 2 audio sources, 2 sensors and $T=8000$.

$434-444$ (1997). DOI 10.1109/78.554307

5. Belouchrani, A., Bourennane, E., Abed-Meraim, K.: A closed form solution for the blind separation of two sources from two sensors using second order statistics. In: European Signal Processing Conferenc (EUSIPCO). Florence, Italy (2006)

6. Comon, P., Jutten, C.: Handbook of Blind Source Separation: Independent Component Analysis and Applications, 1st edn. Academic Press (2010)

7. Durán-Díaz, I., Cruces-Alvarez, S.A.: A joint optimization criterion for blind DS-CDMA detection. EURASIP Journal on Advances in Signal Processing 2007(79248), 1-11 (2007)

8. Hypvarinen, A., Karhunen, J., Oja, E.: Independent Component Analysis. John Wiley and Sons (2001)

9. Itakura, F., Saito, S.: Analysis synthesis telephony based on the maximum likelihood method. In: 6th International Congress on Acoustics, vol. C, pp. 17-20. Tokyo, Japan (1968)

10. Rouxel, A., Guennec, D.L., Macchi, O.: Unsupervised adaptive separation of impulse signals applied to EEG analysis. In: IEEE International Conference on Acoustics, Speech, Signal Processing (ICASSP), vol. 1, pp. 420-423. Istanbul, Turkey (2000) 
11. Varajarajan, V., Krolik, J.: Multichannel system identification methods for sensor array calibration in uncertain multipath environments. In: IEEE Signal Processing Workshop on Statistical Signal Processing (SSP), pp. 297-300. Singapore (2001)

12. Wang, Z., Zhang, X., Cao, T.: Intelligent Control and Automation: International Conference on Intelligent Computing, ICIC 2006 Kunming, China, August 16-19, 2006, chap. A New Blind Source Separation Algorithm Based on Second-Order Statistics for TITO, pp. 29-34. Springer Berlin Heidelberg, Berlin, Heidelberg (2006). DOI 10.1007/978-3-540-37256-1_4 